\section{Two types of fibroblast drive arthritis}

\author{
Fibroblast cells promote the development of rheumatoid arthritis. The finding \\ that two distinct fibroblast populations affect different aspects of the disease in \\ mice has implications for efforts to develop clinical treatments. SEE LETTER P.246
}

\section{THOMAS A. WYNN}

$\mathrm{F}$ ibroblasts are a type of cell found in all tissues of the body. They help to maintain the structural integrity of connective tissues by secreting the protein collagen and other components of the material that comprises the extracellular matrix, which provides support for neighbouring cells ${ }^{1}$. Despite their crucial role in maintaining a healthy tissue architecture, it is becoming increasingly clear that fibroblasts can also contribute to inflammation and tissue injury in a variety of autoimmune diseases, including rheumatoid arthritis - a condition characterized by persistent inflammation, bone erosion and the destruction of cartilage in the joints ${ }^{2}$. On page 246, Croft et al. ${ }^{3}$ report evidence indicating that the progression of rheumatoid arthritis is driven by two populations of fibroblast that have distinct gene-expression profiles, are located in different regions of the joint and contribute to different aspects of the disease.

It was previously unclear whether a single population of fibroblasts contributes to the destruction of joints that is observed in rheumatoid arthritis, or whether different fibroblast types with distinct functional activities are involved, as seems to be the case in other diseases in which fibroblasts have key roles ${ }^{4}$. To study this, Croft and colleagues examined samples of the synovial membrane (layers of cells that surround a joint) from people with rheumatoid arthritis. They found that high expression of the protein FAP $\alpha$ in these tissues was strongly linked with development of the progressive form of the disease.

Studying a mouse model of arthritis, the authors used an imaging technique to track the expression of FAPa in cells $s^{5}$. This showed that higher levels of FAP $\alpha$ expression correlate with greater severity of inflammatory swelling of the ankle joints. Cells expressing FAP $\alpha$ were found to migrate to the bone and cartilage prerequisite behaviour for the cells to cause destruction there.

To characterize the FAP $\alpha$-expressing fibroblasts, the authors used a technique called mass cytometry. This enabled them to identify two distinct populations in the joint (Fig. 1), with one population residing in the synovial membrane in a region called the lining layer (LL), and the other located in the sub-lining layer (SL). Whether or not the protein Thy-1 (also known as CD90) was expressed by these cells offered a way of distinguishing between the two populations, because Thy-1 expression is associated only with cells in the SL. Croft et al. found that both of these cell populations increased in mouse joints when arthritis developed, with the number of fibroblasts in the SL correlating with the severity of inflammation and the number of fibroblasts in the LL being associated with cartilage damage. This provided crucial data indicating that different populations might have roles in distinct aspects of rheumatoid arthritis.

The authors investigated whether there were any other indications that these two cell populations represented unique subsets that have distinct functional roles. Using their mouse model and performing single-cell RNA sequencing ${ }^{6}$ of cells isolated from the synovial membrane at the peak of inflammation (which were isolated in a way that excluded immune cells), the authors identified five cellular groupings that had distinct gene-expression profiles. One group corresponded to the FAP $\alpha$ expressing, Thy-1-lacking cells in the LL and the other four were distinct subpopulations of the FAP $\alpha$ - and Thy-1-expressing cells in the SL. These SL cells expressed many genes that encode signalling molecules called cytokines, which are associated with immune-system function and inflammation. By contrast, the cells in the LL expressed genes associated with cartilage and bone erosion. These distinctive patterns of gene expression suggested that the two cell populations might perform non-overlapping functions in vivo.

To assess the functional role of the fibroblasts, the authors used their mouse model of arthritis ${ }^{5}$ to deplete fibroblasts during the initiation and maintenance phases of the disease. The depletion of all FAP $\alpha$-expressing fibroblasts led to a substantial reduction in the number of cells in the synovial membrane, suggesting a general reduction in inflammation. The loss of FAP $\alpha$-expressing cells also correlated with a reduction in the thickness of the ankle joint compared with that in animals in which fibroblasts had not been depleted.

Strikingly, the reduction in synovial inflammation and joint destruction, which was quantified by assessing the degree of loss of cartilage and bone, was observed whether the fibroblasts were depleted early or late in the course of the disease. This protection from disease progression correlated with a loss of both fibroblast populations, but not with a loss of pericytes, a type of fibroblast found associated with blood vessels. The reduced inflammation was accompanied by a decrease in immune cells such as neutrophils, monocytes

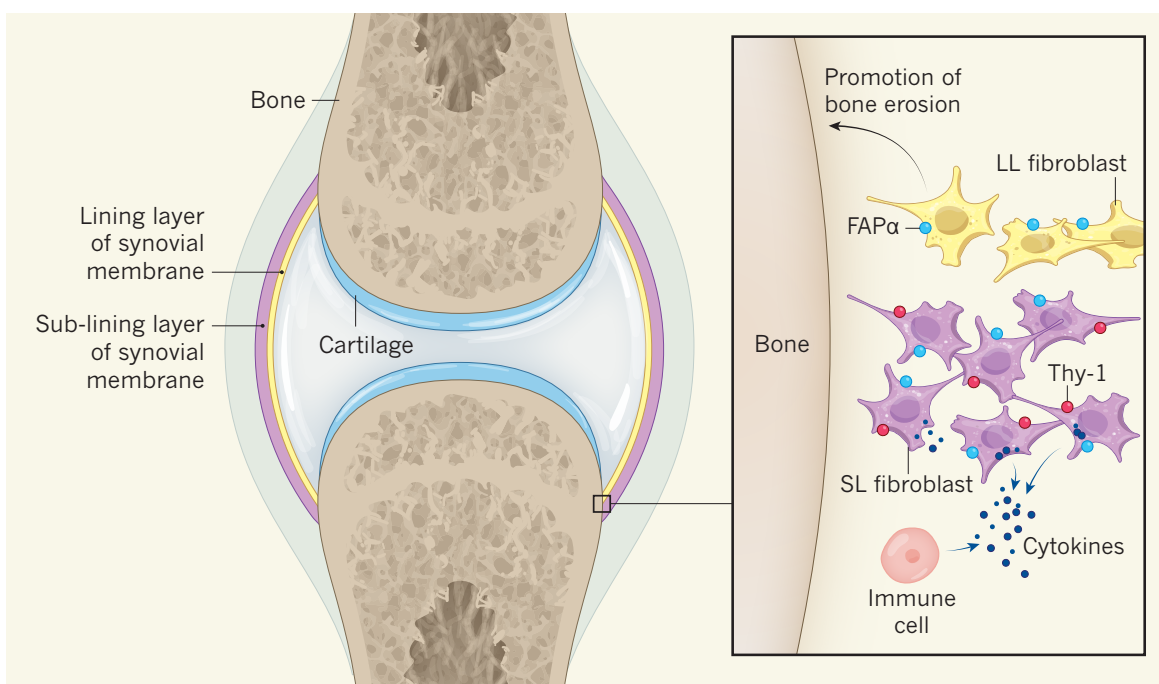

Figure 1 | Two populations of fibroblast have distinct roles in rheumatoid arthritis. Cells called fibroblasts contribute to the development of rheumatoid arthritis ${ }^{2}$, a condition characterized by damage to the joints that is marked by inflammation and the destruction of bone and cartilage. Using a mouse model of the disease and tissue samples from people with rheumatoid arthritis, Croft et al. ${ }^{3}$ report that two distinct fibroblast populations have specific roles in the disease. One population is located in the lining layer (LL) of the synovial membrane (layers of cells that surround a joint). These fibroblasts express the protein FAPa and are associated with bone and cartilage destruction. The other population expresses FAP $\alpha$ and the protein Thy-1, and is found in the synovial membrane's sub-lining layer (SL). These fibroblasts are associated with the inflammation observed in the disease; they express signalling molecules called cytokines that can attract immune cells. 
and macrophages, which are major drivers of inflammation. Interestingly, macrophages in the synovial membrane of FAPa-cell-depleted mice had a pattern of gene expression that is characteristic of an anti-inflammatory state. This raises the question of whether diseaseassociated macrophages are a source of proinflammatory cytokines, and also whether these cells acquire an anti-inflammatory profile when numbers of FAPa-expressing cells are reduced ${ }^{7}$. However, this was not specifically confirmed by the authors.

To test the individual contributions of the two fibroblast populations directly, the authors isolated cells that either expressed or lacked Thy-1, and injected them into the inflamed joints of arthritic mice. Mice that received Thy-1-expressing fibroblasts developed more-severe inflammatory arthritis, but not greater bone or cartilage destruction than was the case for animals that did not receive a cellular transplant. By contrast, injection of fibroblasts lacking Thy-1 did not affect the level of inflammation, but bone erosion was greater than it was in animals that had not received a transplant. The authors concluded that the subset of Thy-1-expressing fibroblasts drove inflammation by producing cytokines, whereas the fibroblast subset lacking Thy-1 contributed to bone and cartilage destruction.

To investigate whether their findings might have relevance for human disease, the authors examined samples of cells from the synovial membrane of people with either rheumatoid arthritis or osteoarthritis - a form of arthritis characterized by joint damage but little or no inflammation ${ }^{8}$. They found that people with rheumatoid arthritis had a larger population of fibroblasts that express FAP $\alpha$ and Thy- 1 than did people with osteoarthritis. Future studies should determine whether fibroblasts that express FAPa but lack Thy-1 are present in greater numbers in the synovial LL of people with rheumatoid arthritis or osteoarthritis than in the LL of healthy people, because this was not specifically examined by the authors, but is predicted by their model.

These exciting findings raise the possibility that clinical strategies might be developed for the selective depletion, targeted replacement or functional conversion of fibroblast subpopulations. Such approaches might one day provide treatment options not just for rheumatoid arthritis, but for a wide range of chronic inflammatory diseases.

Thomas A. Wynn is in the Inflammation and Immunology Research Unit, Pfizer, Cambridge, Massachusetts 02139, USA. e-mail:drtwynn@gmail.com

1. Lynch, M. D. \& Watt, F. M. J. Clin. Invest. 128, 26-35 (2018).

2. Malmström, V., Catrina, A. I. \& Klareskog, L. Nature Rev. Immunol. 17, 60-75 (2017)

3. Croft, A. P. et al. Nature 570, 246-251 (2019).

4. Huelsken, J. \& Hanahan, D. Cell 172, 643-644 (2018).
5. Roberts, E. W. et al. J. Exp. Med. 210, 1137-1151 (2013).

6. Vallejos, C. A., Risso, D., Scialdone, A., Dudoit, S. \& Marioni, J. C. Nature Methods 14, 565-571 (2017).

7. Vannella, K. M. \& Wynn, T. A. Annu. Rev. Physiol. 79, 593-617 (2017)

8. Loeser, R. F., Goldring, S. R., Scanzello, C. R. \&
Goldring, M. B. Arthritis Rheum. 64, 1697-1707 (2012).

The author declares competing financial interests. See go.nature.com/2wrzcpu for details.

This article was published online on 29 May 2019.

\section{ANCIENT GENOMICS}

\section{Human lineages in the far north}

Humans reached the Americas from northeastern Siberia during the last ice age. Genomic analyses of ancient and modern individuals reveal the history of the peoples who have populated these regions. SEE ARTICLE P.182 \& LETTER P.236

\section{ANNE C. STONE}

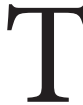
The far northeast of Siberia was the gateway to the Americas for ancient humans, and today is home to diverse cultures whose members speak many languages. During the Late Pleistocene period (the ice age that lasted from about 126,000 to 11,700 years ago), this area of Siberia was connected to North America; the land bridge and adjacent areas formed a region known as Beringia. Hunter-gatherer populations seem to have ranged widely ${ }^{1-3}$ across Siberia and into Beringia, sustained by megafauna such as woolly mammoths, and other animals. In this issue, Sikora et al. ${ }^{4}$ (page 182) and Flegontov et al. ${ }^{5}$ (page 236) examine the genetic footprints of past peoples in northeastern Siberia and northern North America, to work out their relationships to modern communities. Sikora and colleagues also examine how these peoples were affected by climate change over the past 40,000 years.

Sikora et al. analysed genomic data from 34 people from ancient northeastern Siberia. Two individuals were buried at Yana RHS in Russia - a 31,600-year-old archaeological site that contains the earliest human remains found in the far northeast of Siberia - and the others date from 9,800 to 600 years ago. The Yana individuals provide the only genomic data gathered so far from northeastern Siberia before the Last Glacial Maximum (LGM, about 26,500 to 19,000 years ago), although

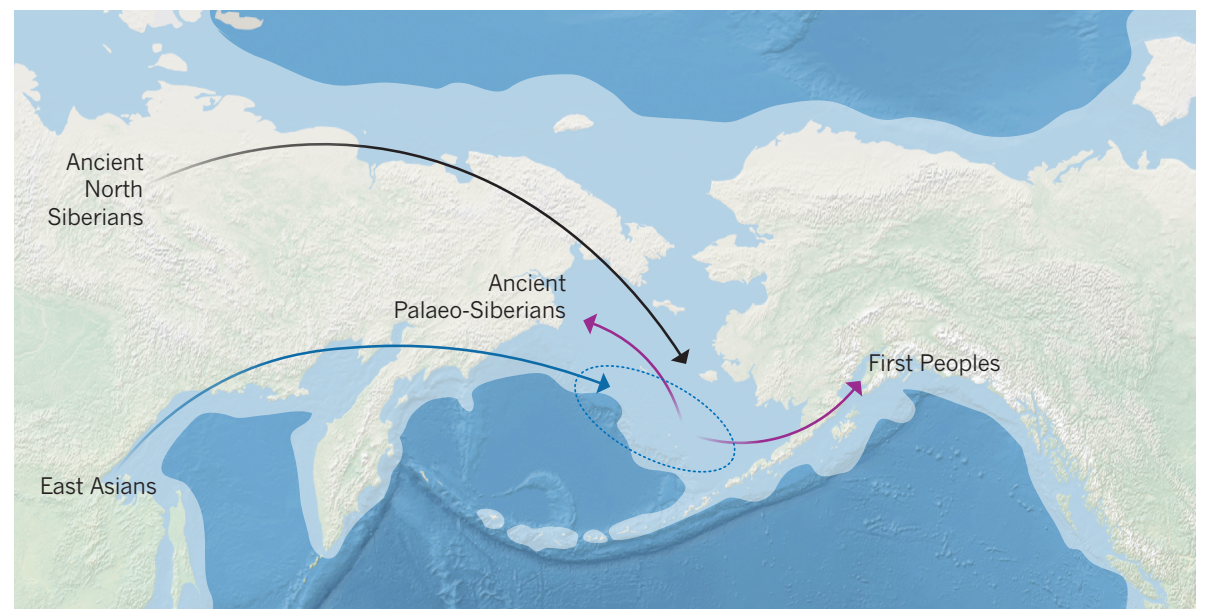

Figure 1 | Migration of ancient peoples across Beringia. During the last ice age, an area of land called Beringia connected Siberia and the Americas. Beringia is shown as the pale blue area superimposed on the modern maps of Siberia and North America. Sikora and colleagues ${ }^{4}$ analysed DNA from the remains of ancient individuals from northeastern Siberia, and suggest that a group they call the Ancient North Siberians probably moved from Siberia to more-hospitable regions, such as southern Beringia (dotted oval), during the Last Glacial Maximum, extending from about 26,500 to 19,000 years ago. These individuals, the authors posit, were ancestral both to the first humans who inhabited the Americas (the First Peoples) and to a subsequent Siberian group (the Ancient Palaeo-Siberians). East Asians also contributed genetic ancestry to these two groups. The Ancient Palaeo-Siberian population subsequently expanded throughout Siberia, whereas the First Peoples expanded into the Americas; the two groups are estimated to have diverged about 24,000 years ago. 